\title{
On stabilizability of the manifold of steady states in a model of the spread of a mutating viruses
}

\author{
Ju. Ermoshkina ${ }^{1}$

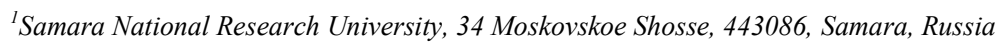

\begin{abstract}
A system of semilinear parabolic equations with a manifold of steady states is considered and the conditions of stabilizability of this manifold are obtained in the paper.
\end{abstract}

Keywords: bifurcation; parabolic equation; the manifold of equilibrium states; the model of interaction of viruses

\section{Introduction}

Consider the system of differential equations:

$$
\begin{gathered}
\frac{d a}{d t}=A(a, y, z), \\
\frac{d y}{d t}=B y+Y(a, y, z), \\
\frac{d z}{d t}=C z+Z(a, y, z),
\end{gathered}
$$

where $a, A \in R^{l} ; y, Y \in R^{k} ; z, Z \in R^{m}$. Assume that $A(a, 0,0) \equiv 0, Y(a, 0,0) \equiv 0, Z(a, 0,0) \equiv 0$. Then the system (1) has a manifold of equilibrium states $\mathfrak{M}=\left\{(a, 0) \mid a \in R^{l}, 0 \in R^{k} \times R^{m}\right\}$.

Following [1,2], let say that the manifold $\mathfrak{M}$ is stable with respect to variable $x=(y, z)$, if for any point $a \in R^{l}$ and any neighborhood of zero $W$ in phase space $R^{k} \times R^{m}$ we can specify such a neighborhood of zero $W_{0} \subset R^{k} \times R^{m}$, that for any point $x_{0}=\left(y_{0}, z_{0}\right) \in W$ the corresponding solution $a=a\left(t, a_{0}, x_{0}\right), x=x\left(t, a_{0}, x_{0}\right)\left(a\left(0, a_{0}, x_{0}\right)=a_{0}, x\left(0, a_{0}, x_{0}\right)=x_{0}\right)$ satisfies the ratio $x=x\left(t, a_{0}, x_{0}\right) \in W$ when $t \geq 0$.

Let say that $\mathfrak{M}$ is asymptotically stable with respect to variable $x=(y, z)$, if it is stable with respect to variable $x$ and, moreover, $\lim _{t \rightarrow \infty} x\left(t, a_{0}, x_{0}\right)=0$ for all $x_{0} \in W$.

Let say that $\mathfrak{M}$ is stabilized, if it is asymptotically stable with respect to variable $x$ and when $t \rightarrow \infty\left\{a\left(t, a_{0}, x_{0}\right)\right.$, $\left.x\left(t, a_{0}, x_{0}\right)\right\}$ converge to some point of diversity $\mathfrak{M}$, if $x_{0} \in W_{0}$.

M.A. Ayzerman and F.R. Gantmakher established that the state of equilibrium of nonholonomic system is stable, if all roots of the characteristic equation, except for the zero roots, the number of which equals the number of equations of nonholonomic connections, have negative real parts $[3,4]$. Each perturbed motion, which is close enough to unperturbed motion, is converge to one of the possible established motions, belong to a given manifold, when $t \rightarrow \infty$. [5]

\section{Model description}

Let consider the model of interaction of two populations of microorganisms in one-dimensional case. This system is based on the equations of Fisher-Kolmogorov-Petrovsky-Piskunov. Let $u(x, t)$ and $v(x, t)$ be concentrations of the two sub-types of a virus at a point $x$ and a time $t$. Consider the problem on the interval $x \in[0 ; 1]$. The system has the form:

$$
\left\{\begin{array}{l}
\frac{\partial u(x, t)}{\partial t}=D_{1} \frac{\partial^{2} u(x, t)}{\partial x^{2}}+a_{1} u(x, t)\left(1-q_{1} v(x, t)\right)(1-u(x, t)-v(x, t)) ; \\
\frac{\partial v(x, t)}{\partial t}=D_{2} \frac{\partial^{2} v(x, t)}{\partial x^{2}}+a_{2} v(x, t)\left(1-q_{2} u(x, t)\right)(1-u(x, t)-v(x, t)),
\end{array}\right.
$$

where $a_{1}, a_{2}$ - the replacement rates for populations $\mathrm{u}$ and $\mathrm{v}$ accordingly, $D_{1}, D_{2}-$ the coefficients of diffusion, $q_{1}$, $q_{2}-$ the coefficients of the interaction between individuals of different populations.

The condition of impermeability at the ends of the considered interval are considered as the boundary conditions in this problem. They look like:

$$
\begin{aligned}
& \left.\frac{\partial u(x, t)}{\partial x}\right|_{x=0}=\left.\frac{\partial u(x, t)}{\partial x}\right|_{x=1}=0 ; \\
& \left.\frac{\partial v(x, t)}{\partial x}\right|_{x=0}=\left.\frac{\partial v(x, t)}{\partial x}\right|_{x=1}=0 .
\end{aligned}
$$

Continuous functions are chosen as the initial conditions. They have the form:

$$
\begin{gathered}
u(x, 0)=\left\{\begin{array}{c}
0,9\left(-5(x-1)^{2}+1\right), u>0, \\
0, u \leq 0
\end{array}\right. \\
v(x, 0)=\left\{\begin{array}{c}
0,9\left(-5 x^{2}+1\right), v>0 \\
0, v \leq 0 .
\end{array}\right.
\end{gathered}
$$




\section{Analysis of the model}

Let find the conditions of stability for the model (2). First of all, let find the equilibrium states of the system. These stationary solutions are obtained by equating all partial derivatives to zero in equations of model. Introduce functions $f_{1}$, $f_{2}$, which defined by the following equations:

$f_{1}=a_{1} \bar{u}\left(1-q_{1} \bar{v}\right)(1-\bar{u}-\bar{v})=0$
$f_{2}=a_{2} \bar{v}\left(1-q_{2} \bar{u}\right)(1-\bar{u}-\bar{v})=0$.

From equations (5) it is easy to obtain the equilibrium states of the system:
$\left(\bar{u}_{1}, \bar{v}_{1}\right)=(0,1)$
$\left(\bar{u}_{2}, \bar{v}_{2}\right)=(1,0)$;
$\left(\bar{u}_{3}, \bar{v}_{3}\right)=(0.5,0.5)$.

To obtain the closest linear system, where $(u, v)$ is close to $(\bar{u}, \bar{v})$, let introduce the infinitesimal perturbations:

$\xi(x, t)=u(x, t)-\bar{u} ; \eta(x, t)=v(x, t)-\bar{v}$.

Consider the approximation of functions $f_{1}(u, v), f_{2}(u, v)$ near any equilibrium states $(\bar{u}, \bar{v})$. Multivariable calculus may be used to obtain the following approximations:

$$
\begin{aligned}
& f_{1}(u, v) \approx f_{1}(\bar{u}, \bar{v})+\frac{\partial f_{1}}{\partial u} \xi+\frac{\partial f_{1}}{\partial v} \eta \\
& f_{2}(u, v) \approx f_{2}(\bar{u}, \bar{v})+\frac{\partial f_{2}}{\partial u} \xi+\frac{\partial f_{2}}{\partial v} \eta .
\end{aligned}
$$

Members of the second and higher orders can be neglected since the perturbations are infinitely small. Taking into consideration equations (5), let receive:

$$
\begin{aligned}
& f_{1}(u, v) \approx \frac{\partial f_{1}}{\partial u} \xi+\frac{\partial f_{1}}{\partial v} \eta \\
& f_{2}(u, v) \approx \frac{\partial f_{2}}{\partial u} \xi+\frac{\partial f_{2}}{\partial v} \eta .
\end{aligned}
$$

Finally, substituting equations determining the perturbations (7) into the equations defining the model (2), leads to a set of equations showing how the perturbance will develop in time:

$$
\begin{aligned}
& \frac{\partial \xi}{\partial t}=D_{1} \frac{\partial^{2} \xi}{\partial x^{2}}+\frac{\partial f_{1}}{\partial u} \xi+\frac{\partial f_{1}}{\partial v} \eta, \\
& \frac{\partial \eta}{\partial t}=D_{1} \frac{\partial^{2} \eta}{\partial x^{2}}+\frac{\partial f_{2}}{\partial u} \xi+\frac{\partial f_{2}}{\partial v} \eta .
\end{aligned}
$$

Let consider the Jacobian matrix for the system (10). The signs of the eigenvalues of this matrix will give the conditions of stability of the stationary solutions.

$$
\mathrm{A}=\left(\begin{array}{ll}
\frac{\partial f_{1}}{\partial u} & \frac{\partial f_{1}}{\partial v} \\
\frac{\partial f_{2}}{\partial u} & \frac{\partial f_{2}}{\partial v}
\end{array}\right)
$$

Accounting that $f_{1}=a_{1} u\left(1-q_{1} v\right)(1-u-v), f_{2}=a_{2} v\left(1-q_{2} u\right)(1-u-v)$, let calculate the partial derivatives of these functions on variables $u, v$. Then, the Jacobian matrix A takes the form:

$$
\mathrm{A}=\left(\begin{array}{ll}
a_{1}\left(1-q_{1} v\right)(1-2 u-v) & a_{1} u\left(q_{1}(u+2 v-1)-1\right) \\
a_{2} v\left(q_{2}(v+2 u-1)-1\right) & a_{2}\left(1-q_{2} u\right)(1-u-2 v)
\end{array}\right)
$$

Following the research conducted by Juan Carlos Cantero and Andrei Korobeinikov [6], consider the position of equilibrium $\left(\bar{u}_{3}, \bar{v}_{3}\right)=(0.5,0.5)$.

Substitute $\left(\bar{u}_{3}, \bar{v}_{3}\right)=(0.5,0.5)$ in $(12)$ :

$$
\mathrm{A}=\left(\begin{array}{ll}
-0.5 a_{1}\left(1-0.5 q_{1}\right) & -0.5 a_{1}\left(1-0.5 q_{1}\right) \\
-0.5 a_{2}\left(1-0.5 q_{2}\right) & -0.5 a_{2}\left(1-0.5 q_{2}\right)
\end{array}\right)
$$

The determinant of the Jacobian matrix equals to zero, and the stability of the solution will depend on the trace of the matrix A. If the trace of the matrix is negative, then the solution is stable. Then if $a_{1} q_{1}+a_{2} q_{2}<2\left(a_{1}+a_{2}\right)$, the stationary solution is stable. And if $a_{1} q_{1}+a_{2} q_{2}>2\left(a_{1}+a_{2}\right)$, the solution is not stable.

\section{Numerical modeling}

To solve the problem (2)-(4) let make an explicit finite-difference scheme. To do this, replace the differential operators of their mesh analogues. Receive:

$$
\left\{\begin{array}{l}
\frac{u_{i}^{k+1}-u_{i}^{k}}{\tau}=D_{1} \frac{u_{i+1}^{k}-2 u_{i}^{k}+u_{i-1}^{k}}{h^{2}}+a_{1} u_{i}^{k}\left(1-q_{1} v_{i}^{k}\right)\left(1-u_{i}^{k}-v_{i}^{k}\right) \\
\frac{v_{i}^{k+1}-v_{i}^{k}}{\tau}=D_{2} \frac{v_{i+1}^{k}-2 v_{i}^{k}+v_{i-1}^{k}}{h^{2}}+a_{2} v_{i}^{k}\left(1-q_{2} u_{i}^{k}\right)\left(1-u_{i}^{k}-v_{i}^{k}\right) .
\end{array}\right.
$$

The boundary conditions will take the form:

$$
\begin{aligned}
& \frac{u_{1}^{k+1}-u_{-1}^{k+1}}{h}=0 ; \\
& \frac{v_{1}^{k+1}-v_{-1}^{k+1}}{h}=0 .
\end{aligned}
$$

Define the initial conditions as follows:

$$
\begin{gathered}
u_{i}^{0}=\left\{\begin{array}{c}
0,9\left(-5\left(x_{i}-1\right)^{2}+1\right), u_{i}^{0}>0, \\
0, u_{i}^{0} \leq 0 ;
\end{array}\right. \\
v_{i}^{0}=\left\{\begin{array}{c}
0,9\left(-5 x_{i}^{2}+1\right), v_{i}^{0}>0, \\
0, v_{i}^{0} \leq 0 .
\end{array}\right.
\end{gathered}
$$

Their graphs are presented in figure1. 


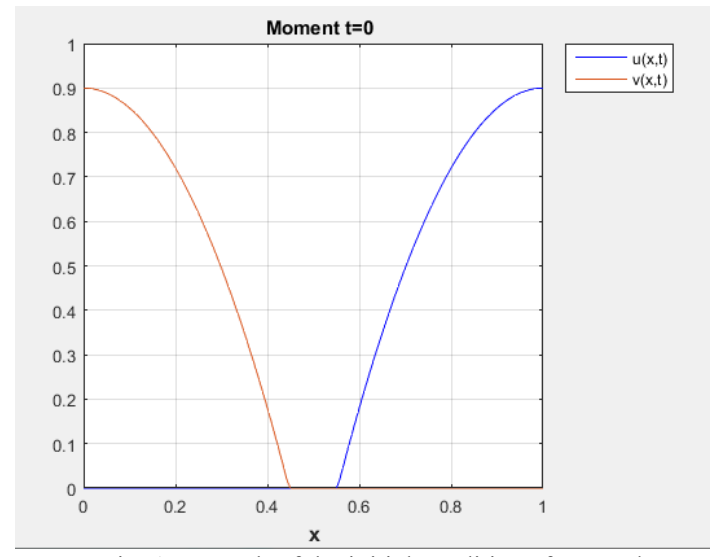

Fig. 1. Graph of the initial conditions for $u$ and $v$

To solve the problem (14)-(16) the program was realised in Matlab, which calculates the values of the grid functions on the time interval $0 \leq \mathrm{t} \leq 600$.

\section{Different cases}

Consider the case when the coefficients of the first and the second equations are equal, i. e. $a_{1}=a_{2}=1, D_{1}=D_{2}=0.001$, $\mathrm{q}_{1}=\mathrm{q}_{2}$. Separating the variables and solving the task on eigenvalues, find the value of parameters $\mathrm{q}_{1}=\mathrm{q}_{2}=2$, in the transition through which the bifurcation happens in the system. To illustrate this phenomenon, consider the three different cases:

1. $\mathrm{q}_{1}=\mathrm{q}_{2}<2$

2. $\mathrm{q}_{1}=\mathrm{q}_{2} \approx 2$

3. $\mathrm{q}_{1}=\mathrm{q}_{2}>2$

In the first case, the trajectories of system converge to the equilibrium $(0,5 ; 0,5)$, belonging to the manifold of equilibrium states of the system. By Ayzerman-Gantmacher's theorem, the state of equilibrium of system is stable. Thus, manifold is stabilized. In the second case, there is a soft loss of stability of the system when passing through the critical value, and in the third case, it is possible to observe a complete loss of stability.

\subsection{Case, when $q_{1}=q_{2}<2$.}

For the first case, when $\mathrm{q}_{1}=\mathrm{q}_{2}=1.5$, the dynamics of function $\mathrm{u}(\mathrm{x}, \mathrm{t})$ is presented in figure 2 . The dynamics of function $\mathrm{v}(\mathrm{x}, \mathrm{t})$ is presented in figure 3 . In figure 4 a solution in a finite time $\mathrm{t}=600$ is presented.

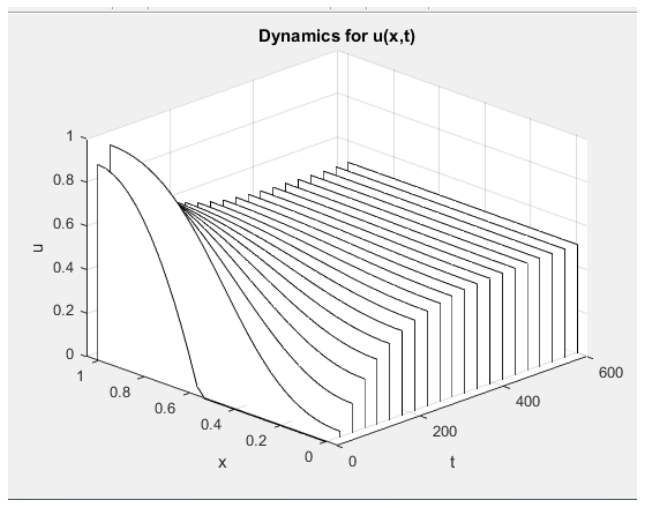

Fig. 2. The dynamics of function $u(x, t)$ for case 1 .

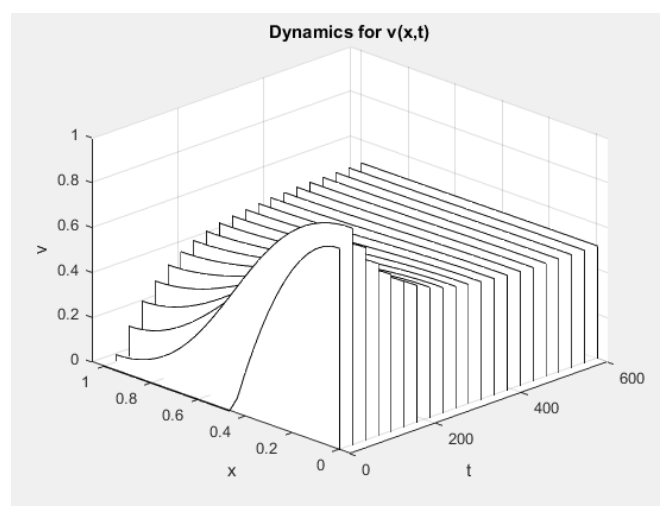

Fig. 3. The dynamics of function $v(x, t)$ for case 1 . 


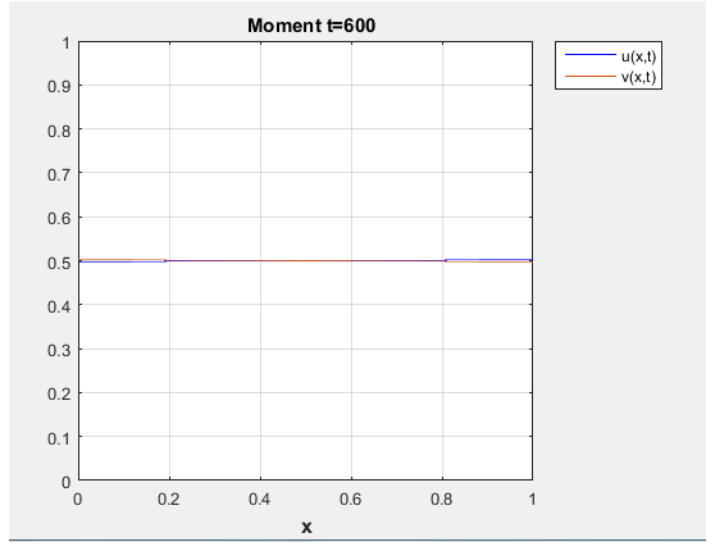

Fig. 4. A solution in a finite time $\mathrm{t}=600$ for case 1 .

\subsection{Case, when $q_{1}=q_{2} \approx 2$.}

For the second case, when $\mathrm{q}_{1}=\mathrm{q}_{2}=2.05$, the dynamics of function $\mathrm{u}(\mathrm{x}, \mathrm{t})$ is presented in figure 5 . The dynamics of function $\mathrm{v}(\mathrm{x}, \mathrm{t})$ is presented in figure 6 . In figure 7 a solution in a finite time $\mathrm{t}=600$ is presented.

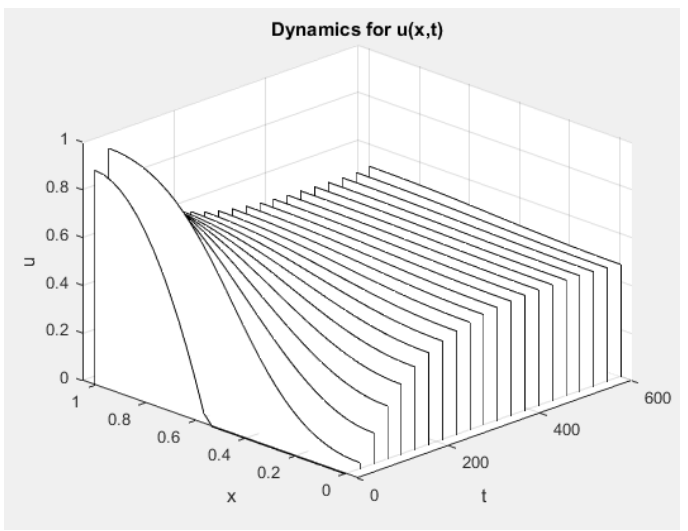

Fig. 5. The dynamics of function $u(x, t)$ for case 2 .

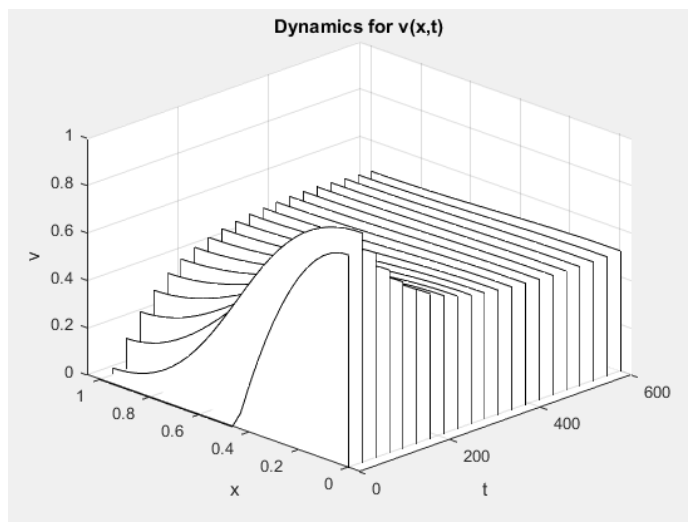

Fig. 6. The dynamics of function $v(x, t)$ for case 2 .

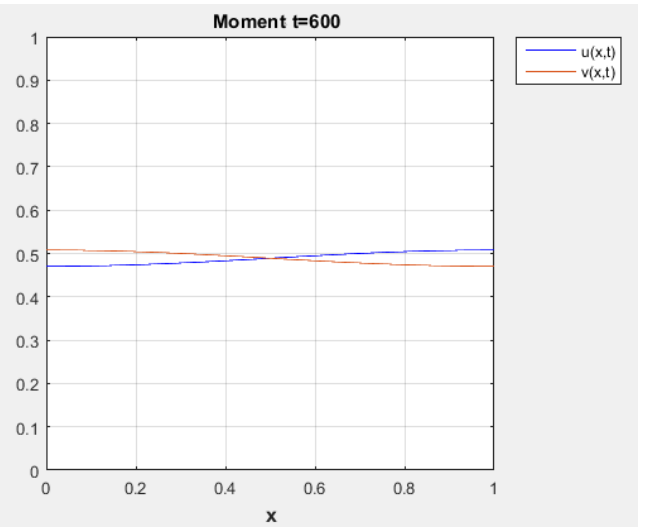

Fig. 7. A solution in a finite time $\mathrm{t}=600$ for case 2 . 
5.3. Case, when $q_{1}=q_{2}>2$.

For case 3 , when $q_{1}=q_{2}=2.5$, the dynamics of function $\mathrm{u}(\mathrm{x}, \mathrm{t})$ is presented in figure 8 . The dynamics of function $\mathrm{v}(\mathrm{x}, \mathrm{t})$ is presented in figure 9 . In figure 10 a solution in a finite time $\mathrm{t}=600$ is presented.

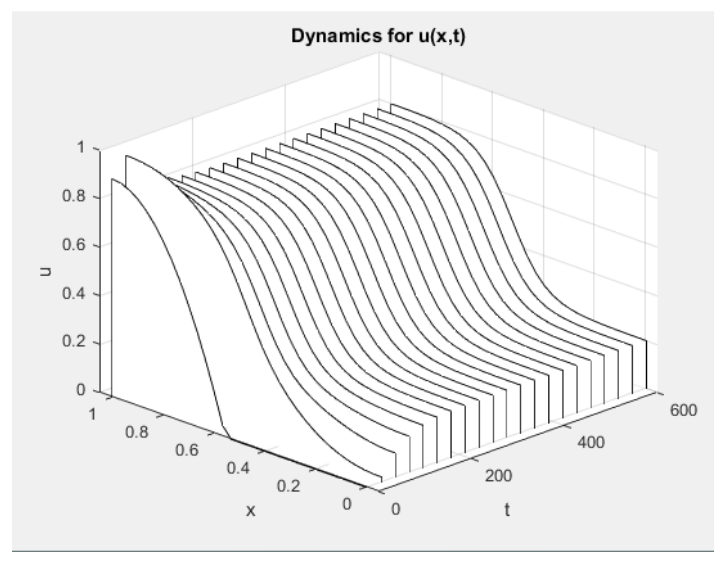

Fig. 8. The dynamics of function $u(x, t)$ for case 3 .

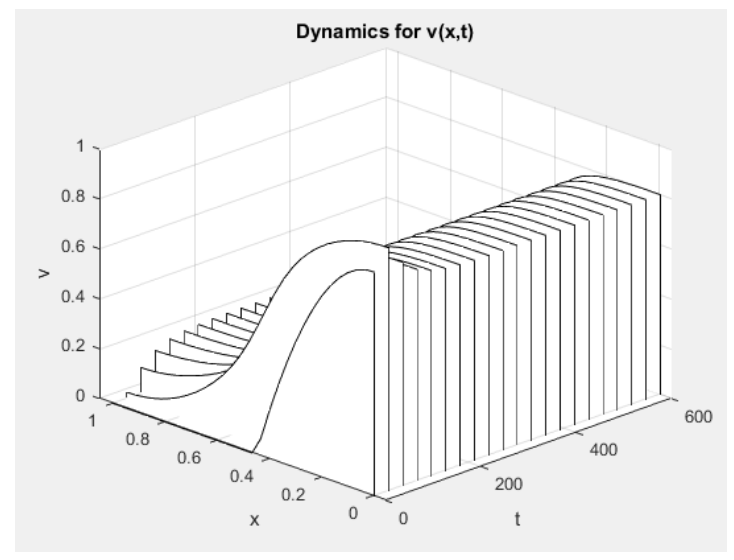

Fig. 9. The dynamics of function $v(x, t)$ for case 3 .

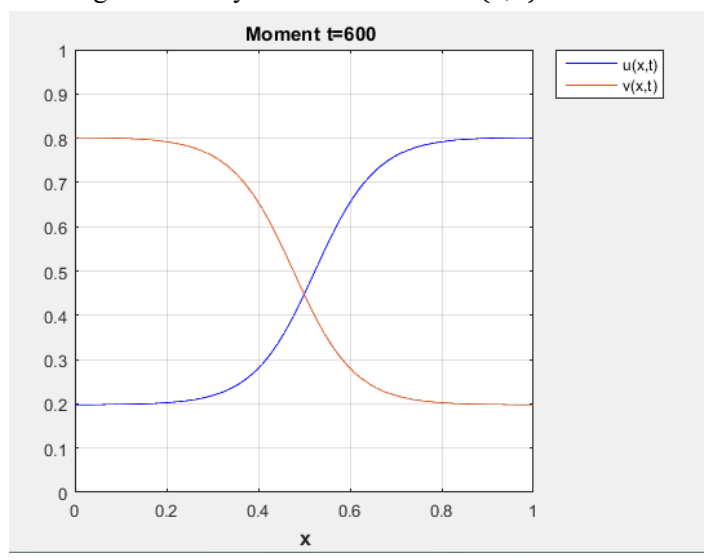

Fig. 10. A solution in a finite time $\mathrm{t}=600$ for case 3 .

\section{Conclusion}

Hence, it is shown that for $\mathrm{q}_{1}=\mathrm{q}_{2}<2$ the manifold of equilibrium states of the system is stabilized, and when passing through the value of the coefficients of the interaction $\mathrm{q}_{1}=\mathrm{q}_{2}=2$ loss of stability occurs in the system.

\section{Acknowledgements}

The paper was supported by the Russian Foundation for Basic Research and the government of the Samara region in the framework of a research project № 16-41-630529. 


\section{References}

[1] Strygin VV, Sobolev VA. Razdelenie dvizheniy metodom integral'nykh mnogoobraziy. Moscow: Nauka, 1988; 256 p.

[2] Strygin VV, Sobolev VA. Effect of geometric and kinetic parameters and energy dissipation on orientation stability of satellites with double spin. Cosmic Research 1976; 14: 331-335.

[3] Ayzerman MA, Gantmakher FR. Stabilität der Gleichgewichtslage im einem night-holonomen System. Z. angew. Math und Mcch. 1957; 37(1/2):74-75.

[4] Neymark YuI, Fufaev NA. Dinamika negolonomnykh sistem. Moscow: Nauka, 1967; 520 p.

[5] Kalenova VI, Karapetyan AV, Morozov VM, Salmina MA. Negolonomnye mekhanicheskie sistemy i stabilizatsiya dvizheniya. Fundamental'naya i prikladnaya matematika 2005; 11: 7: 117-158.

[6] Cantero JC, Korobeinikov A. The spread of Two Viral Strains on a Plant Leaf. Workshop on Virus Dynamics and Evolution.

[7] Murray JD. Lectures on Nonlinear Differential-Equation Models in Biology. Oxford: Clarendon Press, 1977; 379 p.

[8] Murray JD. Mathematical Biology I. An Introduction. New York: Springer, 2001; 576 p. 